\title{
The Influence of the Magnetic Field and Ultraviolet Waves to the Liquid Fillers for Liquid Lense
}

\author{
Liena Yu. Vergun \\ Molecular Physics Department, Faculty of Physics, Taras Shevchenko National University of Kyiv, Ukraine
}

\begin{abstract}
The question about properties of liquid fillers for liquid lenses under actions of magnetic field and ultraviolet waves are investigated. The liquid model system such as glycerol is used. The coefficients transmission and coefficients of temperatures increasing are experimentally determined. It is shown that the presence of constant magnetic field and UV-waves stabiliz formation of a wall layer. So the vibrational and rotary motions of molecules and its clusters are stabilized in liquid fillers. It is assumed that the chromatic aberration can be associated with the formation and redistribution of particles in the surface layer at the interface between the solid and liquid phases.
\end{abstract}

Key words: Glycerol, magnetic field, ultraviolet waves.

\section{Introduction}

It is known liquid lenses are used in optoelectronic systems telescopes $[1,2]$. The operating conditions of such devices connected with the interaction of UV radiation and constant magnetic field. For increasing of the quality of liquid lenses pay attention to the properties of liquid filler for exclusion of chromatic aberration [3, 4]. The determination of action UV-waves and constant magnetic field in model system is proposed for possible reasons of aberration. It is known glycerol is the simplest composition of liquid filler for liquid lenses [5]. The appearance of feature molecular structure in glycerol under the influence of a magnetic field and UV-radiation is demonstrated in work [6].

The nature of the transmission of light waves after constant magnetic field action and action of UV-waves is experimentally determined. Time of action these external factors is corresponded to relaxation time in the system [7]. The transmission coefficients of glycerol after action constant magnetic field and UF-waves are obtained.

Corresponding author: Liena Yu. Vergun, Ph.D., research fields: magnetic field, defects in crystal like structures.
The value of a magnetic field was $22.4 \mathrm{mT}$ [6]. In view of Ref. [8] it has been suggested that the occurrence of chromatic aberration is due to the relaxation processes in the boundary layer of fluid components. To exclude the impact of these processes on the image quality the author offered to hold preliminary processing liquid fillers under UV-waves and constant magnetic field.

\section{Materials and Method}

The type of glycerol with GOST technical standards $6259-75$ is used. The scheme of laboratory bench is represented in Fig. 1a (where 1-working cell of KFK2MP, 2-glass cuvette, 3-glycerol, 4-UF emitter, 5-Helmholtz coils, 6-electronic scoreboard). The view of working cell of KFK2MP (photoelectric colorimeter) with Helmholtz coils is represented in Fig. $1 \mathrm{~b}$.

\section{Results and Discussion}

The dependences of the transmission coefficient $\tau(\lambda)$ are shown in Fig. 2. In this figure by number " 1 "- the experimental datas of glyserol without action of the external factors, by number " 2 "- the experimental data for glyserol after action UV-waves (time of action 


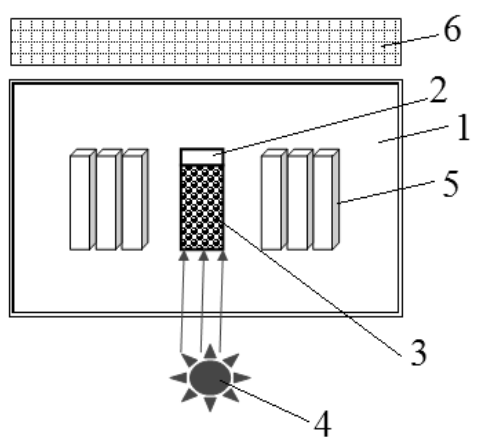

(a)

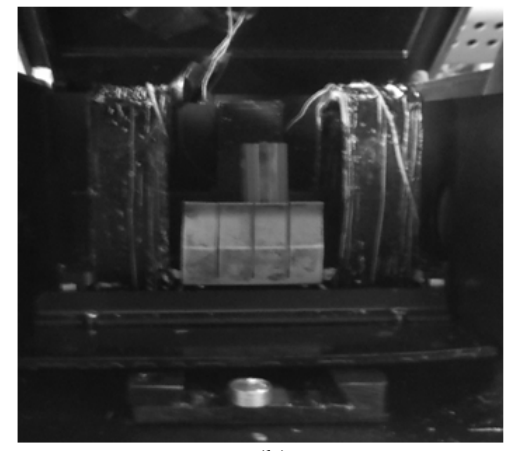

(b)

Fig. 1 The scheme (a) and view (b) of laboratory bench.

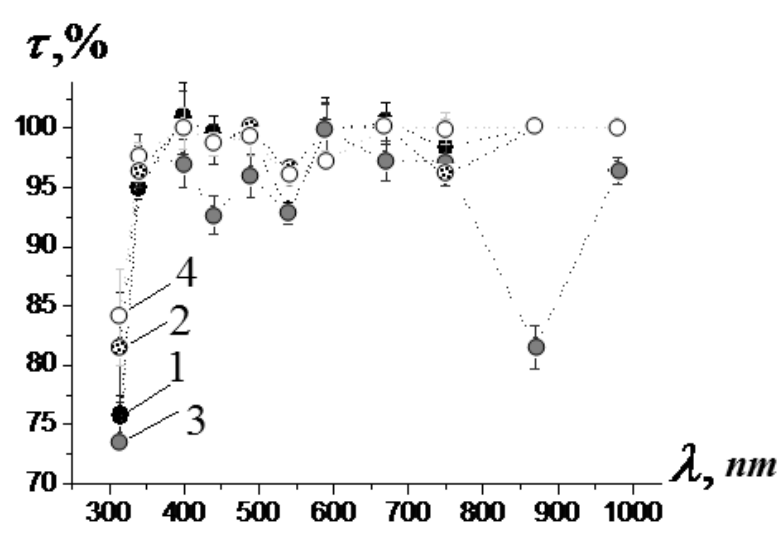

Fig. 2 The dependence $\tau(\lambda)$.

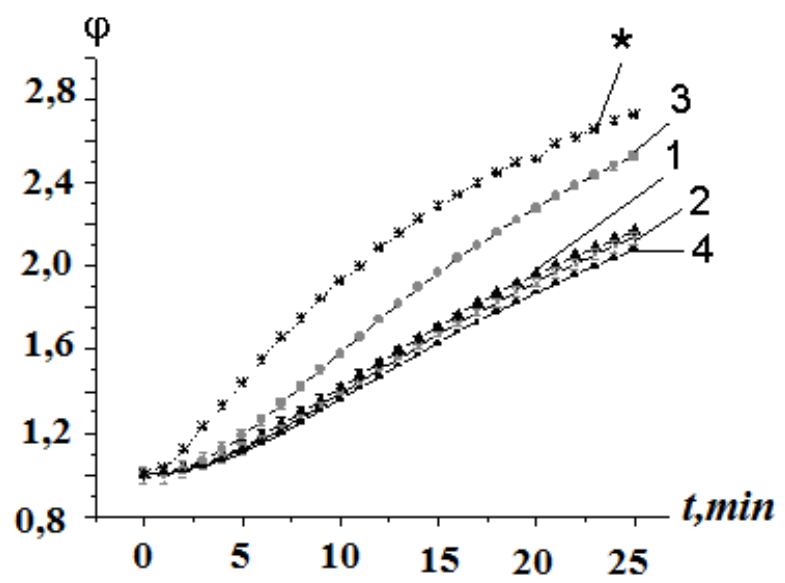

Fig. 3 The dependence $\varphi(t)$.

15 minutes), by number " 3 " - the experimental data of glyserol after action constant magnetic field (time of action 15 minutes), by number " 4 " - the experimental data after action UV-waves and constant magnetic field (time of action 15 minutes) are indicated.

As shown in Fig. 3 the joint action of UV waves and constant magnetic field decreases the difference between values of coefficients transmission in the area of wavelengths from $380 \mathrm{~nm}$ (violet color) to $750 \mathrm{~nm}$ (red color). The obtained results indicate a decrease in the probability of the appearance of chromatic aberration.

According to Ref. [8], the phenomenon of chromatic aberration can be associated with the formation and redistribution of particles in the surface layer at the interface between the solid and liquid phases. The redistribution of particles and their clusters occurs at dissimilarities of velocity of their displacements at change of the action of external factors [9, 10]. An experiment was conducted for this purpose. A cuvette with the substance (glycerol) was placed in the heat-insulating container. The temperature sensor was located in the center of the cuvette. The temperature sensor readings were recorded in increments of minute. Fig. 3 shows the dependence of the dimensionless coefficient $\varphi$ (the coefficient of temperatures increasing) on time $t$. The coefficient $\varphi$ was calculated according to the formula $\varphi=T_{i} / T_{0}$, where $T_{0}$ is the temperature of the measurement at the initial moment, $T_{i}$-the temperature in the following intervals of time.

The following symbols are introduced on the graph: In Fig. 3, by number " 1 "- the experimental data of glycerol without the action of the external factors, by number " 2 "- the experimental data of glycerol after the action of UV-waves (time of action 15 minutes) by number " 3 " - the experimental data of glycerol after 
the action of the constants, magnetic field (time of action 15 minutes), by number " 4 "- - the experimental data after action of UV-waves and constant magnetic field (time of action 15 minutes). The symbol "** denotes the experimental data of the standard substance $\left(\mathrm{Al}_{2} \mathrm{O}_{3}\right)$.

As can be seen from Fig. 3 at treatment of glycerol by constant magnetic field and UV irradiation the smallest increasing of temperature is observed. The experimental data obtained correspond to the experimental results shown in Fig. 2. According to our opinion, the treatment of UV iiradiation with a magnetic field leads to a uniform distribution of molecules both in the surface layer near the wall and in the entire volume.

\section{Conclusions}

Experimental studies of the optical and thermophysical properties of a model system of a liquid component for liquid lenses (glycerin) under the influence of UV irradiation and a magnetic field were conducted. It is shown that the presence of constant magnetic field and UV-waves stabiliz formation of a wall layer. So the vibrational and rotary motions of molecules and its clusters are stabilized in liquid fillers.

\section{References}

[1] Gibson, B. K. 1991. "Liquid Mirror Telescope History."
Journal of the Royal Astronomical Society of Canada 85 (4): 158-71.

[2] Berge, B. 2005. "Liquid Lense Technology: Principle of Electrowetting Based Lenses and Applications to Imaging." IEEE, 227-30.

[3] Stallinga, S., Vrehen, J., Wals, J., Stapert, H., and Verstegen, E. 2000. "Liquid Crystal Aberration Compensation Devices." Proceedings of SPIE 48: 50-9.

[4] Vergun, L.Y. 2015. "The Using of Magnetic Field for the Improvement of Properties of Liquid Fillers in a Liquid Lense." In Abstracts XXII Galyna Puchkovska International School-Seminar Spectroscopy of Molecules and Crystals, 155.

[5] Calo, E., and Khutoryanskiy, V. V. 2015. "Biomedical Application of Hydrogels: A Reviews of Patents and Commercial Products." European Polymer Journal 65: 252-67.

[6] Pelizzeti, E., and Schiavello, M. 1991. Photochemical Conversion and Storage of Solar Energy. Springer Science \& Business Media.

[7] Kaneoke, Y., Furuse, M., and Yoshida, K. 1989. "Transfer Index of MR Relaxation Enhancer: A Quantitative Evaluation of MR Contrast Enhancemen.” AJNR Am J Neuroradiol 10 (2): 329-33.

[8] Angell, C. A., and Ngai, K. L. 2000. "Relaxation in Glass-Forming Liquids and Amorphys Solids." Journal of Applied Physics 88 (6): 3113-57.

[9] Root, L. J., and Stillinger, F. H. 1989. "Short-Range Order in Glycerol: A Molecular Dynamics Study." J. Chern. Phys. 90 (2): 1200-08.

[10] Milner, A. A., Korobenko, A., Flo, J., Sh. Averbukh, I., and Milner, V. 2015. "Magneto-Optical Properties of Paramagnetic Superrotors.” Phys. Rev. Lett. 115 (3): 033005. 